\title{
WBSN in IoT Health-Based Application: Toward Delay and Energy Consumption Minimization
}

\author{
Ahmed Alkhayyat $\mathbb{D}^{1},{ }^{1}$ Ahmed A. Thabit, ${ }^{2}$ Fahad A. Al-Mayali, ${ }^{1}$ and Qammer H. Abbasi ${ }^{3}$ \\ ${ }^{1}$ Department of Computer Technical Engineering, College of Technical Engineering, Islamic University, 54001 Najaf, Iraq \\ ${ }^{2}$ Department of Communications Computer Engineering, Al-Rafidain University, 10062 Baghdad, Iraq \\ ${ }^{3}$ School of Engineering, University of Glasgow, G12 8QQ Glasgow, UK
}

Correspondence should be addressed to Ahmed Alkhayyat; ahmedalkhayyat85@gmail.com

Received 27 December 2018; Accepted 10 March 2019; Published 8 May 2019

Academic Editor: Ghufran Ahmed

Copyright (c) 2019 Ahmed Alkhayyat et al. This is an open access article distributed under the Creative Commons Attribution License, which permits unrestricted use, distribution, and reproduction in any medium, provided the original work is properly cited.

\begin{abstract}
The wireless body sensor network (WBSN) technologies are one of the essential technologies of the Internet of things (IoT) growths of the healthcare paradigm, where every patient is monitored through a group of small-powered and lightweight sensor nodes. Thus, energy consumption is a major issue in WBSN. The major causes of energy wastage in WBSN are collisions and retransmission process. However, the major cause of the collision happened when two sensors are attempting to transmit data at exactly the same time and same frequency, and the major cause of the retransmission process happened when the collision takes place or data does not received properly due to channel fading. In this paper, we proposed a cognitive cooperative communication with two master nodes, namely, as two cognitive master nodes (TCMN), which can eliminate the collision and reduce the retransmission process. First, a complete study of a scheme is investigated in terms of network architecture. Second, a mathematical model of the link and outage probability of the proposed protocol are derived. Third, the end-to-end delay, throughput, and energy consumption are analyzed and investigated. The simulation and numerical results show that the TCMN can do system performance under general conditions with respect to direct transmission mode (DTM) and existing work.
\end{abstract}

\section{Introduction}

1.1. Introduction to IoT Health-Based Application. Now, IoTs are one of the most powerful communication standards of the 21 st century. In the IoT environment, all objects in our daily life become part of the Internet due to their communication and computing capabilities. IoT spreads the thought of the Internet and makes it much universal. IoT lets all-inone communications among various kinds of electronic devices $[1,2]$. Hence, the IoTs have become more creative in some fields, for example, healthcare technologies. In healthcare technologies, IoTs involved numerous types of inexpensive sensors, wearable and implanted, that allow old persons to enjoy current medical healthcare services everyplace, any time, and it improved old persons' quality of life [3-7]. In this work, we proposed new architectures of the
IoT health-based application that consists of five stages which depicted in Figure 1. Each stage of this architecture is further explained in more detail as follows.

(1) The WBSN Stage (Stage 1). In this stage, some sensors are placed on the human body sewed into fabric (wearable sensors) or implanted under the skin (inbody sensors). These sensors can be electroencephalogram (EEG), electrocardiogram (ECG), electromyography (EMG), or blood pressure measuring sensors. The data recorded by the sensors are sent to the master node (MN) via wireless 802.15.6 standard, then MN transfers data to the local processing unit (such as smart phone, tablet, and computer) through wireless medium, and then local processing unit (LPU) forwards the data to the next stage 


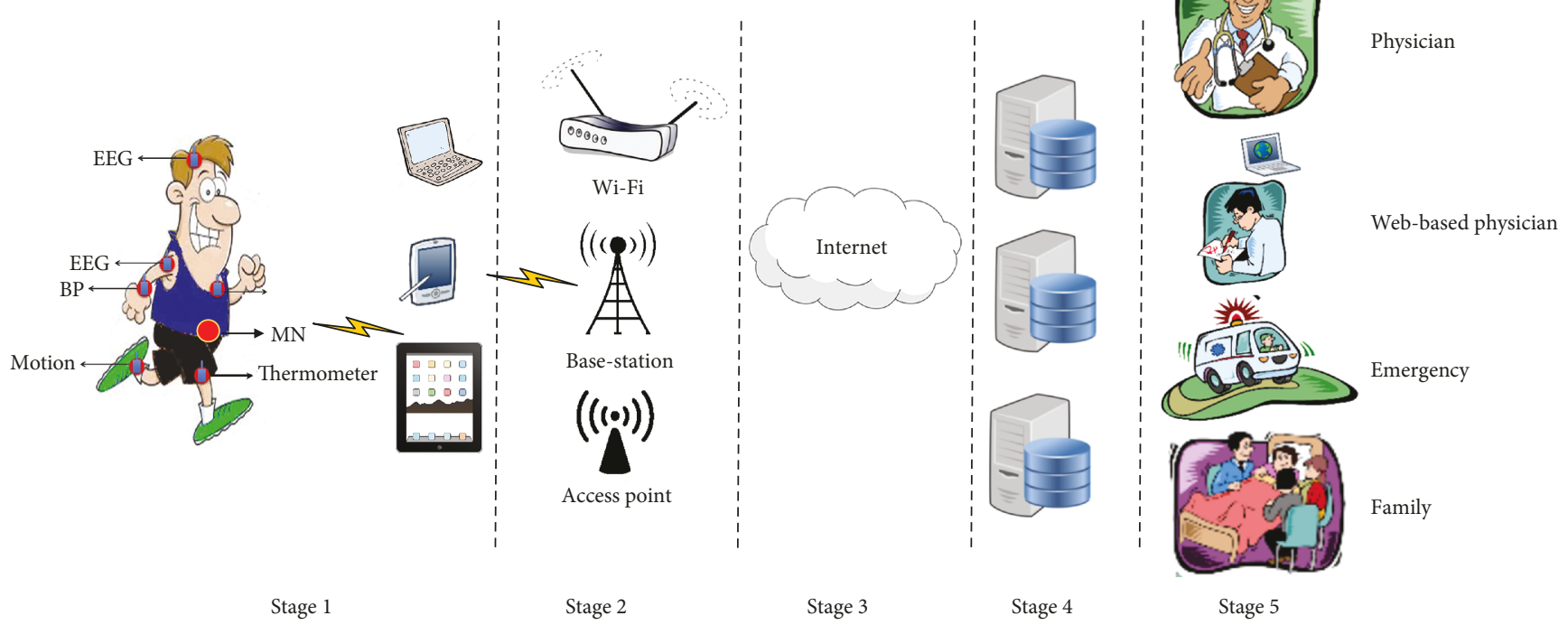

FIgURE 1: WBSN in IoT health-based application.

(2) The Bridge Stage (Stage 2). In this stage, the data transfer from stage 1 to stage 2 over one of the selected wireless communication technologies which could be access point, Wi-Fi, or cellular base station. Stage 2 represents as the bridge to connect the WBSN to the infrastructure Internet and sometimes located within room or outdoor. Collected data from this stage is required to be transferred to stage 4 in order to be stored and analyzed for the final destination

(3) The Internet Stage (Stage 3). In this stage, bridge the gap between stage 2 and stage 4 via either exiting wireless technology or guided medium such as fiber optics

(4) Data Storage and Analyzing Stage (Stage 4). In this stage, the data is stored and analyzed to make final decision where the data have to go or it should kept at stage 4

(5) Healthcare Service Stage (Stage 5). In this stage, it provides healthcare services to patients. The data may be transferred to doctors or hospitals and transferred to intermediate family

In this work, we enhance the IoT health-based application at stage 1 . Sensors in WBSN utilize wireless medium to exchange their data, where existence of the human body could disturb the transmission between sensors over wireless medium, resulting to a definite and special radio propagation that should be appropriately considered for in the design of algorithms in the WBSN systems. In addition, the necessity for efficient power consumption shall be addressed through power control solutions due to repeated battery replacing not practical, which is a very hard job for implanted sensor node $[8,9]$.

One of the most suitable layers to deal with IoT healthbased WBSN system challenges is the physical layer. In the physical layer, there are many techniques which can enhance the network performance, such as modulations, channel coding, channel estimation, network coding, and virtual diversity $[10,11]$. In this paper, we are interested in the latter one (virtual diversity) because it is considered as a plug and play technique. Virtual diversity can be achieved through cooperative communication; the on-body sensors work together to deliver the data efficiently to the MN. There is a rich literature on cooperative communication in different network systems, but a few work has been done in the IoT health-based WBSN systems.

With the fast deployment of wireless facilities for the last decade, the radio spectrums have become a valued and limited resource. Furthermore, the Federal Communications Commission has reported that most of the licensed spectrum is roughly underutilized. As a promising method, a cognitive radio (CR) is proposed to address the dilemma between spectrum shortage and spectrum underutilization. CR lets unlicensed users to access licensed slots or subchannels while taking into account the effect of interference on the licensed users. Thus, it should not exceed the tolerable interference threshold [12]. Therefore, the underlying approach is utilized, where the unlicensed users are allowed to utilize the licensed slots or subchannels with only a limited transmission power to prevent the interference of unlicensed users from exceeding the interference threshold that the licensed user can tolerate [13].

1.2. Literature Review. To date, a few number of researches have been performed on the cognitive cooperative communication for WBSN. Jingling et al. [14] have made an attempt to enhance the capacity of the WBSN system through CR. Nazir and Sabah [15] studied a hybrid power control and resource block allocation game for the cooperative wireless BAN. The cost function for WBSN is derived and used to analyze the optimal power allocation on assigned resource blocks. 
Chavez-Santiago et al. [16] proposed a CR-based solution for a medical BAN (MBAN), and then they studied and investigated the proposed method over the PHY and MAC layers. The proposed method is relied on the utilizing of ultrawideband (UWB) with IEEE 802.15.6 standard. Furthermore, the cognitive capabilities are applied in the BNC utilizing two UWB modalities. Syed and Yau [17] presented two architectures and two state-of-the-art applications of CR (i.e., electromagnetic interference (EMI) reduction as well as QoS improvement). Januszkiewicz [18] proposed a simplified human body system that is utilized for radio channel discovering in BSN. The objective of the proposed model is used in measurements of cognitive radio channel parameters. Shaikh and Tamil [19] proposed a telemedicine platform architecture consisting of three tiers. The first tier consists of BSN with a gateway, and the communication technology used in this tier is UWB. The cognitive radio controller and the gateway integrated together form the next tier of the architecture. The third tier of the architecture is the communication link between the mobile vehicle and the hospital, which refer to the cognitive radio-enabled communication model. The proposed platform improved the performance discovering free spectrum in both urban and suburban environments. Mahbub et al. [20] analyzed the outage probability using decode and forward cognitive relaying over Rician fading channels, with respect to an interference temperature limit and distance between primary and secondary nodes. Bhandari and Moh [21] addressed a survey on MAC protocols for cognitive radio body sensor networks (CRBSNs). Then, they compared different MAC protocols with one another and discussed the challenging and open issues in the relevant research. Bhatia and Kumar [22] proposed a technique for selecting the best network from the available networks relying on the QoS necessities for various WBSN applications. The proposed system is efficient in creating a best network selection in situations where there is a conflict between different QoS requirements for different WBSN applications. Sodagari et al. [23] presented a review on CRBSNs, specifically for medical applications. They provided a summary of the current systems on interference mitigation for the coexistence of various devices involving wireless body area networks. Further, they studied current energy-efficient MAC methods for CRBSNs. Furthermore, the challenges and difficulties of performing spectrum sharing methodology for WBSNs are investigated. The comparison of the state of the art is shown in Table 1.

Nevertheless, the works in [14-23] have not investigated the collected data nature that shown to be an important issue in IoT health-based network. It also noted that the works did not take into account the quality of service, reliability, and delay; they are the main factors that determine the performance of the IoT health-based WBSN systems. In addition, the interference constraint in the underlying approach has not been considered for CRBSNs.

To address the previous-mentioned issues and facilitate cognitive cooperative communication in WBSN, we proposed two cognitive master nodes (TCMN) based on the IEEE 802.15.6 CSMA policy. The contributions of this work are summarized as follows:
(1) We propose two master node architectures with cognitive cooperative communication which involve the master nodes in cooperation; thus, the on-body sensors are not involved in cooperation as traditional cooperative communication does

(2) In addition, master nodes are involved in the spectrum sensing and selecting free available spectrum which are considered a major cause of the battery consumption in WBSN; thus, the on-body sensors are not involved in the spectrum sensing as traditional CRBSNs do

(3) The proposed protocol supports multiple data (traffics), critical and normal traffics; critical traffics are transmitted over cognitive cooperative communication and normal traffics are transmitted over cognitive direct transmission

(4) We reveal that the TCMN can enhance the endto-end delay, throughput, and energy consumption compared to direct 802.15.6 standard and existing work

The rest of the paper is outlined as follows: system model and architecture and the new proposed architecture are presented in Section 2. Section 3 investigates the new architecture under the licensed spectrum and describes the proposed protocol. Then, modeling and formulating signal-tonoise ratio, outage probability, and critical data index of TCMN in detail are described in Section 3. In Section 4, e2e delay, throughput, and energy consumption of TCMN are inspected, formulated, and analyzed. Simulation and numerical results are addressed in Section 5. Finally, Section 6 draws the conclusion and future work.

\section{WBSN Network Architecture}

First traditional WBSN network architecture is shown in Figure 1. Several sensors homogeneously distributed on/off the human body to look up the health signs, and each of the sensor collects and sends data to the MN. Thus, WBSN is a single-hop topology; all the nodes forward gathered information to the MN. The MN then transmits what was received from the sensors to the LPU; the MN either analyzed the obtained information or retransmitted it over wireless medium to the next stage as shown in Figure 1. In the WBSNs, it must take into account the number of physical environments, because the WBSN is organized on or in the body. In addition, the nodes attached to the body will be mobile-owning human body mobility. Thus, the distances among the sensors and $\mathrm{MN}$ are variable. Therefore, several sensors may be far away from the MN; therefore, delivering data appropriately to the $\mathrm{MN}$ directly is difficult.

Then, in Figure 2(b), a new WBSN network architecture is designed, two masters-slave network architecture. One of the MNs is fixed on the human body, and it is carried on the belt, named as master node 1 (MN1). The other master node (which is shown in Figure 1 as LPU) works as a monitoring node and can receive data sent by the sensor as MN1 can, 
TABLE 1: Comparison of the state-of-the-art work.

\begin{tabular}{|c|c|c|c|c|}
\hline Ref. no. & Proposed protocol & Problem addressed & Compared with & Limitation \\
\hline$[14]$ & $\begin{array}{c}\text { Cyclostationarity-based } \\
\text { sensing (CBS) }\end{array}$ & $\begin{array}{l}\text { (i) Detection probability } \\
\text { (ii) Detection efficiency }\end{array}$ & $\begin{array}{c}\text { Energy detector-based } \\
\text { sensing (EDBS) }\end{array}$ & $\begin{array}{l}\text { (i) IEEE } 802.15 .6 \text { standard is not studied } \\
\text { (ii) Reliability is missing } \\
\text { (iii) QoS is not considered } \\
\text { (iv) Energy efficiency } \\
\text { (v) Collected data nature is not delivered } \\
\text { efficiently } \\
\text { (vi) Mobility is not supported } \\
\text { (vii) Delay is not analyzed } \\
\text { (viii) Both interference and power } \\
\text { constraint are missing }\end{array}$ \\
\hline$[15]$ & $\begin{array}{l}\text { A hybrid power control } \\
\text { and resource block } \\
\text { allocation game }\end{array}$ & (i) Energy saving & Non-CR & $\begin{array}{l}\text { (i) IEEE } 802.15 .6 \text { standard is not studied } \\
\text { (ii) Reliability is missing } \\
\text { (iii) QoS is not considered } \\
\text { (iv) Collected data nature is not delivered } \\
\text { efficiently } \\
\text { (v) Mobility is not supported } \\
\text { (vi) Both interference and power constraint } \\
\text { are missing }\end{array}$ \\
\hline$[16]$ & $\begin{array}{l}\text { CR-based solution for a } \\
\text { MBAN }\end{array}$ & (i) Survey & - & - \\
\hline [17] & $\begin{array}{l}\text { Cognitive radio-based } \\
\text { wireless body area networks }\end{array}$ & (i) Survey & - & - \\
\hline [18] & $\begin{array}{l}\text { A cognitive radio-based } \\
\text { telemedicine system }\end{array}$ & $\begin{array}{l}\text { (i) Probability of missed- } \\
\text { detection (PMD) } \\
\text { average power } \\
\text { (i) Probability of false } \\
\text { alarm (PFA) }\end{array}$ & Non-CR & $\begin{array}{l}\text { (i) IEEE } 802.15 .6 \text { standard is not studied } \\
\text { (ii) Reliability is missing } \\
\text { (iii) QoS is not considered } \\
\text { (iv) Collected data nature is not delivered } \\
\text { efficiently } \\
\text { (v) Mobility is not supported } \\
\text { (vi) Both interference and power constraint } \\
\text { are missing }\end{array}$ \\
\hline [19] & $\begin{array}{c}\text { A cognitive radio body sensor } \\
\text { network (CRBSN) }\end{array}$ & (i) Survey & - & - \\
\hline$[20]$ & CRBAN & $\begin{array}{l}\text { (i) Network capacity } \\
\text { (ii) Spectral efficiency }\end{array}$ & - & $\begin{array}{l}\text { (i) IEEE } 802.15 .6 \text { standard is not studied } \\
\text { (ii) Reliability is missing } \\
\text { (iii) Energy efficiency was not studied } \\
\text { (iv) Collected data nature is not delivered } \\
\text { efficiently } \\
\text { (v) Mobility is not supported } \\
\text { (vi) Delay is not analyzed }\end{array}$ \\
\hline [21] & $\begin{array}{l}\text { Multiple attribute decision- } \\
\text { making (MADM) methods }\end{array}$ & $\begin{array}{l}\text { (i) Network selection } \\
\text { ranking }\end{array}$ & - & $\begin{array}{l}\text { (i) IEEE } 802.15 .6 \text { standard is not studied } \\
\text { (ii) Reliability is missing } \\
\text { (iii) Energy efficiency } \\
\text { (iv) Collected data nature is not delivered } \\
\text { efficiently } \\
\text { (v) Mobility is not supported } \\
\text { (vi) Delay is not analyzed } \\
\text { (vii) Both interference and power constraint } \\
\text { are missing }\end{array}$ \\
\hline [23] & $\begin{array}{l}\text { CR-enabled body } \\
\text { area networks }\end{array}$ & (i) Survey & - & - \\
\hline
\end{tabular}

and it is denoted as the master node 2 (MN2). Due to the mobility of the human body, the distances among the sensor and two MNs vary, where several sensors may be located at a short distance toward the outer $\mathrm{MN}$, while large distance toward the on-body $\mathrm{MN}$ and vice versa. Thus, several sensors may have better channel quality with respect to the MN2 than to the MN1. The new network architecture can significantly move the resending process from the sensors to the MNs compared to the traditional cooperative communication. Thus, new architectures could reduce delay and reduce contention between the sensors which could achieve better energy saving. 


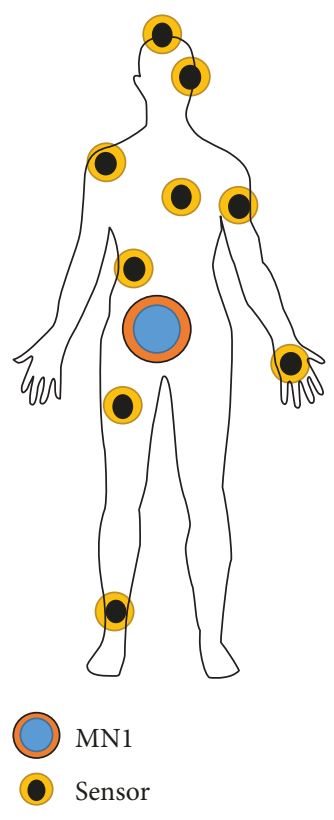

(a)

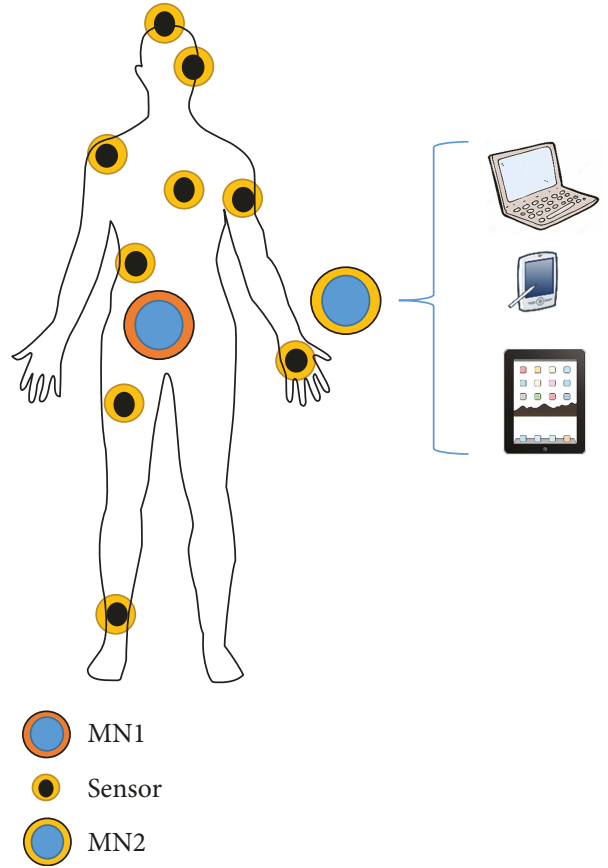

(b)

Figure 2: (a) Traditional WBSN network architecture. (b) Proposed WBSN network architecture.

In Figure 3, two master node architectures in the licensed spectrum are designed. The cellular networks represent the primary network, and the WBSN represents the secondary network that delivers the data utilizing DF method. The CRBSN sensors utilize the underlying method of spectrum sharing with the primary users, i.e., simultaneous primary and secondary transmissions take place only if the interference produced by the secondary user at the primary receivers is under some tolerable threshold.

The advancement in electronics, wireless communications, and integrated circuits has allowed the development of transceiver, which leads to have a multiple transceiver on the single sensor node. In CRBSN, master nodes regularly search for the available spectrum to estimate spectrum availability. Nevertheless, such regular search significantly maximizes the energy consumption of energy-limited system powered via batteries [24]. Consequently, duty of the spectrum sensing is assigned to the master nodes instead of sensors, because the master nodes are embedded with bigger batteries and are replaceable.

In the CRBSN, the primary source (PS) transmits data to the primary receiver (PR), and over the same frequency band, a secondary source sensor (SS) sends a data to a secondary destination, such as MN2 (in this paper, the final destination node is MN2). While MN1 acts as a secondary relay, MN1 helps the SS in sending the data to MN2. The objective of utilizing the licensed spectrum by on-body sensors and master nodes is to avoid the collision due to transmission at the same time and the same frequency.

In what follows, we denoted the channel coefficient of the data links between SS to MN2, SS to MN1, and MN1 to MN2 as $h_{\mathrm{s}, \mathrm{mn} 2}, h_{\mathrm{s}, \mathrm{mn} 1}$, and $h_{\mathrm{mn} 1, \mathrm{mn} 2}$, respectively; in addition, we denoted the channel coefficient of the interference links from SS to PR, PS to MN1, PS to MN2, and MN1 to PR as $h_{\mathrm{s}, \mathrm{r}}$, $h_{\mathrm{p}, \mathrm{mn} 1}, h_{\mathrm{p}, \mathrm{mn} 2}$, and $h_{\mathrm{mn} 1, \mathrm{r}}$, respectively.

\section{Formulation of the Proposed Protocol}

In this section, the model of the links between sensors and master nodes is provided, and then based on the link model, the outage probability is analyzed and investigated over underlay cognitive cooperative communication and incorporating with analysis the normal and critical traffics.

3.1. Link and Outage Probability Analysis. In CRBSN, the sensor (SS) and master node (MN1) must adapt their transmit powers below an interferance threshold so that they should not create any interference for the primary receiver (PR). Simultaneously, since PS, SS, and MN1 are sharing the same channel, transmit power of PS also causes interfarence to the WBSN network. Thus, the minimum transmit power of secondary SS and MN1 can be expressed as follows:

$$
\begin{gathered}
P_{\mathrm{ss}}=\frac{I_{\mathrm{s}, \mathrm{r}}}{h_{\mathrm{s}, \mathrm{r}}} \\
P_{\mathrm{mn} 1}=\frac{I_{\mathrm{mn} 1, \mathrm{r}}}{h_{\mathrm{mn} 1, \mathrm{r}}}
\end{gathered}
$$

where $P_{\mathrm{ss}}$ and $P_{\mathrm{mn} 1}$ are the maximum transmit powers of SS and MN1, respectively; $I_{\mathrm{s}, \mathrm{r}}$ and $I_{\mathrm{mn} 1, \mathrm{r}}$ are interference threshold from SS to PR and interference threshold from 


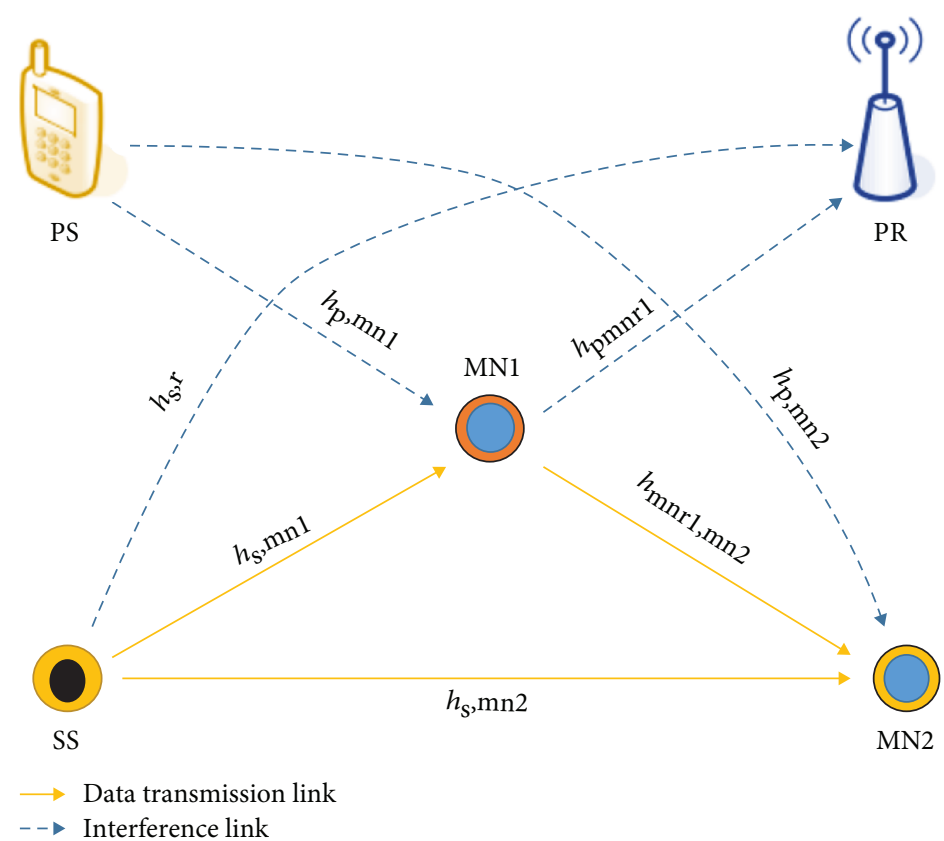

FIgURe 3: Two master nodes under license spectrum.

PS to MN1, respectively. As a result, the signal-to-noise ratio at $\mathrm{MN} 2$ can be expressed as follows:

$$
\gamma_{\mathrm{s}, \mathrm{mn} 2}=\frac{P_{\mathrm{ss}} h_{\mathrm{s}, \mathrm{mn} 2}}{P_{\mathrm{N}}+\left(P_{\mathrm{ps}} h_{\mathrm{p}, \mathrm{mn} 2}\right)},
$$

where $P_{\mathrm{ps}}$ and $P_{\mathrm{N}}$ are the transmission powers of the PS and noise power, respectively. Similarly, the signal-tonoise ratio at MN1 due to transmission from SS and the signal-to-noise ratio at $\mathrm{MN} 2$ due to transmission from MN1 are expressed, respectively, as follows:

$$
\begin{aligned}
\gamma_{\mathrm{s}, \mathrm{mn} 1} & =\frac{P_{\mathrm{ss}} h_{\mathrm{s}, \mathrm{mn} 1}}{P_{\mathrm{N}}+\left(P_{\mathrm{ps}} h_{\mathrm{p}, \mathrm{mn} 1}\right)}, \\
\gamma_{\mathrm{mn} 1, \mathrm{mn} 2} & =\frac{P_{\mathrm{mn} 1} h_{\mathrm{mn} 1, \mathrm{mn} 2}}{P_{\mathrm{N}}+\left(P_{\mathrm{ps}} h_{\mathrm{p}, \mathrm{mn} 2}\right)} .
\end{aligned}
$$

The outage probability from node $i$ to node $j$ can be expressed as follows:

$$
\begin{aligned}
P^{\text {out }} & =P_{\mathrm{r}}\left(R_{i, j} \leq R_{\mathrm{o}}\right) \\
& =P_{\mathrm{r}}\left(\log _{2}\left(1+\gamma_{i, j}\right)<R_{\mathrm{o}}\right) \\
& =P_{\mathrm{r}}\left(h_{i, j}<\frac{2^{R_{\mathrm{o}}}-1}{\widehat{\gamma_{i, j}}}\right) \\
& =1-\exp \left(-\frac{2^{R_{\mathrm{o}}}-1}{\widehat{\gamma_{i, j}}}\right),
\end{aligned}
$$

where $\gamma_{i, j}=h_{i, j} \widehat{\gamma_{i, j}}$. Hence, the channel gain $h_{i, j}$ is an exponential distributed random variable with the mean value $E\left[\left|h_{i, j}\right|^{2}\right]=d_{i, j}^{-\rho}$, where $d_{i, j}^{-\rho}$ is the distance between nodes $i$ and $j$, and $\rho$ is the path loss exponent. The outage probability of the incremental cooperative communication of the two master node-slave architectures can be expressed as follows:

$$
P_{\mathrm{coop}}^{\text {out }}=P_{\mathrm{s}, \mathrm{mn} 2}^{\text {out }} P_{\mathrm{s}, \mathrm{mn} 1}^{\text {out }}+\left(1-P_{\mathrm{s}, \mathrm{mn} 2}^{\text {out }}\right) P_{\mathrm{s}, \mathrm{mn} 1}^{\text {out }} P_{\mathrm{mn} 1, \mathrm{mn} 2}^{\text {out }}
$$

With the help of (6), we can obtain (7) as follows:

$$
\begin{aligned}
P_{\text {coop }}^{\text {out }}= & \left(1-\exp \left(-\frac{2^{R_{\mathrm{o}}}-1}{\widehat{\gamma_{\mathrm{s}, \mathrm{mn} 2}}}\right)\right)\left(1-\exp \left(-\frac{2^{R_{\mathrm{o}}}-1}{\widehat{\gamma_{\mathrm{s}, \mathrm{mn} 1}}}\right)\right) \\
& +\exp \left(-\frac{2^{R_{\mathrm{o}}}-1}{\widehat{\gamma_{\mathrm{s}, \mathrm{mn} 2}}}\right)\left(1-\exp \left(-\frac{2^{R_{\mathrm{o}}}-1}{\widehat{\gamma_{\mathrm{s}, \mathrm{mn} 1}}}\right)\right) \\
& \cdot\left(1-\exp \left(-\frac{2^{R_{\mathrm{o}}}-1}{\widehat{\gamma_{\mathrm{mn} 1, \mathrm{mn} 2}}}\right)\right)
\end{aligned}
$$

Inserting (4), (5), and (6) in (8), we obtain the outage probability under interference constraint.

3.2. Multiple Traffic Transmissions over TCMN. Incremental cooperative communication with multiple traffics for WBSN is proposed in our previous work [25], which discussed a transmission selection issue to support both normal and critical traffics. However, cognitive cooperative communications with multiple traffics are not considered in [25]. In this paper, we continue to study the incremental cooperative communication with multiple traffics for WBSN to improve the throughput and energy consumptions. The TCMN supports multiple traffics, such as critical and normal; thus, the successful transmission probability of multiple traffics using TCMN, then, can be expressed as follows: 


$$
P_{\mathrm{TCMN}}=P(X)+P(Y)
$$

where $P(X)$ is a critical data event. The critical data is sent either over a DTM, that represented by SS to MN2, or over CTM, that represented by SS to MN1, then MN1 to MN2. $P(Y)$ is a normal data event and it is over DTM. Thus, $P(X$ ) expressed as follows:

$$
P(X)=\mathrm{CI} \cdot P_{\text {coop }}^{\mathrm{s}}
$$

where $P_{\text {coop }}^{\mathrm{s}}=\left(1-P_{\text {coop }}^{\text {out }}\right)$ is the successful transmission probability of the CTM, and CI is the critical data index and is expressed as follows:

$$
\mathrm{CI}=1-\left|\frac{\xi_{\min }-\xi_{\max }}{\xi_{\max }}\right|,
$$

where $\xi_{\max }$ is the maxmum critical data index $(=7)$, which represents highest user priority index [26]; $\xi_{\min }$ is the predefined value and it is the minimum critical data index that changes from 0 to 7 . Consequently, CI is changed from 0 to 1 , where $\xi_{\min }$ depends on the collected data from the human body; if the data is critical, then $\xi_{\min }$ is high and converse. Plugging (8) and (11) in (10), we can obtain $P(X)$ as follows:

$$
\begin{aligned}
P(X)= & 1-\left|\frac{\xi_{\min }-\xi_{\max }}{\xi_{\max }}\right|\left(1-\left[\left(1-\exp \left(-\frac{2^{R_{\mathrm{o}}}-1}{\widehat{\gamma_{\mathrm{s}, \mathrm{mn} 2}}}\right)\right)\left(1-\exp \left(-\frac{2^{R_{\mathrm{o}}}-1}{\widehat{\gamma_{\mathrm{s}, \mathrm{mn} 1}}}\right)\right)\right.\right. \\
& \left.\left.+\exp \left(-\frac{2^{R_{\mathrm{o}}}-1}{\widehat{\gamma_{\mathrm{s}, \mathrm{mn} 2}}}\right)\left(1-\exp \left(-\frac{2^{R_{\mathrm{o}}}-1}{\widehat{\gamma_{\mathrm{s}, \mathrm{mn} 1}}}\right)\right)\left(1-\exp \left(-\frac{2^{R_{\mathrm{o}}}-1}{\widehat{\gamma_{\mathrm{mn} 1, \mathrm{mn} 2}}}\right)\right) ?\right]\right]
\end{aligned}
$$

In the same way, the normal data event $P(Y)$ is given as follows:

$$
P(Y)=(1-\mathrm{CI}) \cdot\left(1-P_{\mathrm{s}, \mathrm{mn} 2}^{\text {out }}\right)
$$

Plugging (13) and (12) in (9), we obtain TCMN probability.

\section{Delay and Energy Consumption Analysis of TCMN}

4.1. Delay Formulation and Analysis of TCMN. Generally and traditionally, the average e2e delay, $T_{\mathrm{e} 2 \mathrm{e}}$, of the TCMN will not include the average contention time due to collision because sensors use different spectrums. Thus, average e2e delay is expressed as follows:

$$
T_{\mathrm{e} 2 \mathrm{e}}=T_{\text {suc }}+T_{\text {fail }}
$$

where $T_{\text {suc }}$ is the average successful time with no collision and no fading, and then it is expressed as follows:

$$
\begin{aligned}
T_{\mathrm{suc}}= & \left(\mathrm { CI } \left(T_{\mathrm{act}}^{\mathrm{s}, \mathrm{mn} 2} P_{\mathrm{s}, \mathrm{mn} 2}^{\mathrm{out}}+\left(T_{\mathrm{act}}^{\mathrm{s}, \mathrm{mn} 2}+T_{\mathrm{act}}^{\mathrm{mn} 1, \mathrm{mn} 2}\right)\right.\right. \\
& \left.\left.\cdot\left(1-P_{\mathrm{s}, \mathrm{mn} 2}^{\text {out }}\right) P_{\mathrm{s}, \mathrm{mn} 1}^{\text {out }} P_{\mathrm{mn} 1, \mathrm{mn} 2}^{\text {out }}\right)\right) \\
& +\left((1-C I) P_{\mathrm{s}, \mathrm{mn} 2}^{\text {out }} T_{\mathrm{act}}^{\mathrm{s}, \mathrm{mn} 2}\right) .
\end{aligned}
$$

Equation (15) comprises of double terms. The first term is e2e required time of the transmission when the collected data by the SS were critical, while the second term is e2e required time of the transmission when the collected data by the SS were not critical. It is clear from (15) that as the
CI approaches to " 1 ," then either $T_{\text {act }}^{\mathrm{s}, \mathrm{mn} 2}$ is the required time for sending the data or $T_{\mathrm{act}}^{\mathrm{s}, \mathrm{mn} 2}+T_{\mathrm{act}}^{\mathrm{mnl}, \mathrm{mn} 2}$ is the required time for sending data to the destination. However, as the CI approaches to " 0 ," then $T_{\text {act }}^{\mathrm{s}, \mathrm{mn} 2}$ is the required time for sending data to the destination. $T_{\text {act }}^{\mathrm{mn} 1, \mathrm{mn} 2}$ is the RF activity time of serving the on-body sensor data. Consequently, the average failure time due to fading can be expressed as follows [27]:

$$
\begin{aligned}
T_{\mathrm{fail}}= & \left(P_{\mathrm{s}, \mathrm{mn} 2}^{\text {out }} P_{\mathrm{s}, \mathrm{mn} 1}^{\text {out }}\left(1-P_{\mathrm{mn} 1, \mathrm{mn} 2}^{\text {out }}\right) T_{\mathrm{act}}^{\mathrm{s}, \mathrm{mn} 2}\right) \\
& +\left(\left(T_{\mathrm{act}}^{\mathrm{s}, \mathrm{mn} 2}+T_{\mathrm{act}}^{\mathrm{mn} 1, \mathrm{mn} 2}\right) P_{\mathrm{s}, \mathrm{mn} 2}^{\text {out }} P_{\mathrm{mn} 1, \mathrm{mn} 2}^{\text {out }}\left(1-P_{\mathrm{s}, \mathrm{mn} 1}^{\text {out }}\right)\right. \\
& \left.+P_{\mathrm{s}, \mathrm{mn} 2}^{\text {out }} P_{\mathrm{s}, \mathrm{mn} 1}^{\text {out }} P_{\mathrm{mn} 1, \mathrm{mn} 2}^{\text {out }} T_{\mathrm{act}}^{\mathrm{mn} 1, \mathrm{mn} 2}\right) .
\end{aligned}
$$

Moreover, $T_{\text {act }}$ is the RF activity time, which can be expressed as follows [28]:

$$
T_{\text {act }}=T_{\text {on }}+T_{\text {data }}+T_{\text {ACK }}+2 T_{\alpha} \text {, }
$$

where $T_{\text {data }}$ is the time required to send data packet, and then it is expressed as follows:

$$
T_{\text {data }}=T_{\mathrm{P}}+T_{\mathrm{PHY}}+T_{\mathrm{MAC}}+T_{\mathrm{BODY}}+T_{\mathrm{FCS}} .
$$

The transmission time required for ACK can be expressed as follows:

$$
T_{\mathrm{ACK}}=T_{\mathrm{P}}+T_{\mathrm{PHY}}+T_{\mathrm{MAC}}+T_{\mathrm{FCS}}
$$

The transmission rate of the PHY, MAC headers, and payload are depending on the channel condition between 


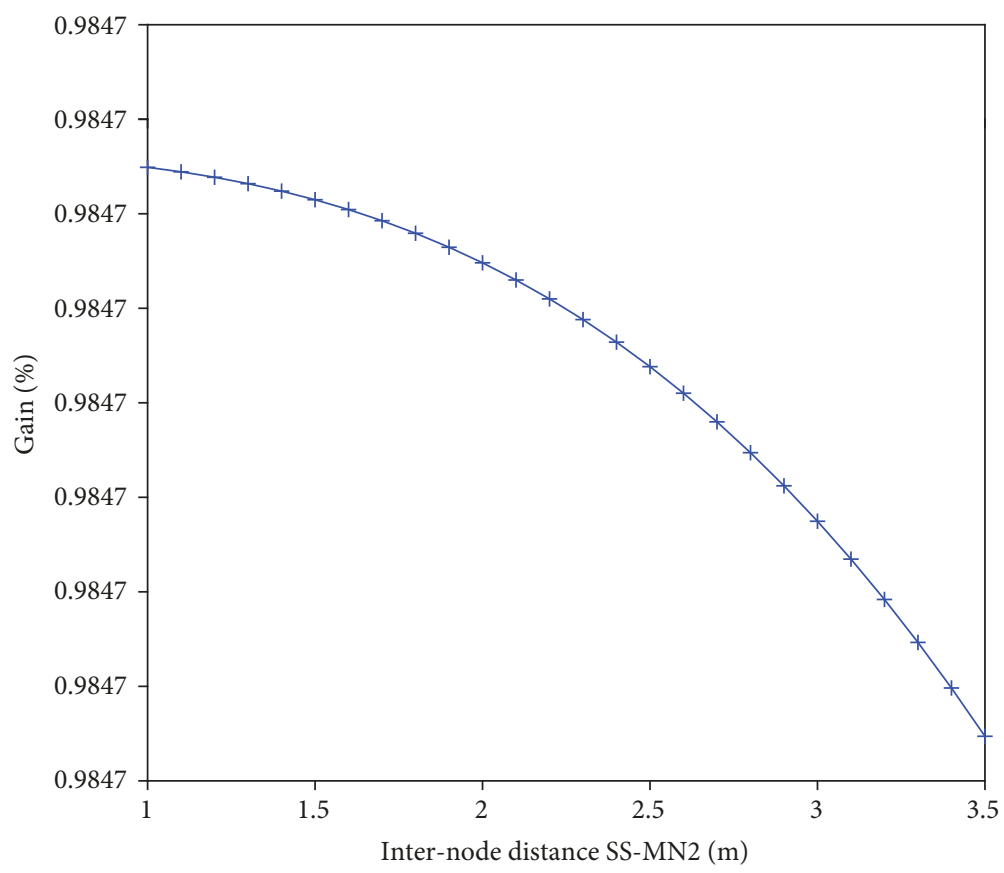

FIgURE 4: TCMN gain with two master nodes. The $I_{\text {thd }}$ is $-3 \mathrm{~dB}$ and $\rho=3$.

nodes [27], where $R_{\text {ate }}$ of the TCMN is given as follows:

$$
R_{\mathrm{ate}}=\mathrm{CI} \cdot P_{\mathrm{coop}}^{\mathrm{s}} R_{\max }+(1-\mathrm{CI}) P_{\mathrm{s}, \mathrm{mn} 2}^{\mathrm{out}} R_{\mathrm{max}},
$$

where $R_{\max }$ is the maximum data rate of IEEE 802.15.6 standard and it is $75.9 \mathrm{Kbps}$.

4.2. TCMN Gain Evaluation. To compare the achievable gain of two master node architectures using license spectrum in the underlying approach, we define the achievable gain $G_{\text {ain }}$ as the reduction in the delay of WBSN with two master nodes utilizing license spectrum. In what follows, $G_{\text {ain }}$ can be expressed as follows:

$$
G_{\mathrm{ain}}=\frac{T_{\mathrm{e} 2 \mathrm{e}}^{\mathrm{old}}-T_{\mathrm{e} 2 \mathrm{e}}^{\mathrm{new}}}{T_{\mathrm{e} 2 \mathrm{e}}^{\mathrm{old}}},
$$

where $T_{\mathrm{e} 2 \mathrm{e}}^{\text {new }}$ is the e2e delay of the proposed protocol and is given in (14), and $T_{\mathrm{e} 2 \mathrm{e}}^{\mathrm{old}}$ is the e2e delay of traditional incremental cooperative communication [28], which is expressed as $T_{\mathrm{e} 2 \mathrm{e}}^{\mathrm{old}}=T_{\text {suc }}+T_{\text {fail }}+T_{\mathrm{c}}$, where $T_{\mathrm{c}}$ is the time due to collision. With the above definition, $G_{\text {ain }}=0$ means that the delay of WBSN with two master nodes by utilizing license spectrum and traditional incremental cooperative communication is the same, while $G_{\text {ain }}=1$ means that delay reduced doubles compared to traditional incremental cooperative communication.

The distance between on-body nodes is chosen randomly; in addition, the distance from the on-body nodes to primary nodes is also chosen randomly. As expected and shown in Figure 4, the gain of the proposed protocol is related to the distance between SS and MN2. Based on the results shown, we can derive the conclusion that TCMN is

\begin{tabular}{|c|c|}
\hline Bandwidth (KHz) & 75.9 \\
\hline Maximum transmission rate $\left(R_{\max }\right)(\mathrm{Kbps})$ & 75.9 \\
\hline Transmission power $\left(P_{\mathrm{t}}\right)$ of sensors $(\mathrm{mW})$ & 3 \\
\hline \multicolumn{2}{|l|}{ Transmission power $\left(P_{\mathrm{ps}}\right)$ of PS $(\mathrm{mW})$} \\
\hline Noise power $\left(P_{\mathrm{N}}\right)(\mathrm{dB})$ & -70 \\
\hline Threshold transmission rate $\left(R_{\mathrm{o}}\right)(\mathrm{b} / \mathrm{s} / \mathrm{Hz})$ & 1 \\
\hline Modulation & DPSK \\
\hline Payload size (bits) & 2000 \\
\hline Packet length $(L)$ (bits) & 2208 \\
\hline Minimum contention windows $\left(\mathrm{CW}_{\min }\right)$ (slots) & 16 \\
\hline Maximum contention windows $\left(\mathrm{CW}_{\max }\right)$ (slots) & 64 \\
\hline Clear channel assessment (bits) & 63 \\
\hline MAC header (bits) & 56 \\
\hline MAC footer (bits) & 16 \\
\hline Short interframe spacing time $\left(T_{\mathrm{pSIFS}}\right)(\mu \mathrm{s})$ & 50 \\
\hline Preamble (bits) & 88 \\
\hline Slot time $\left(T_{\mathrm{s}}\right)(\mu \mathrm{s})$ & 125 \\
\hline Delay time $\left(T_{\alpha}\right)(\mu \mathrm{s})$ & 1 \\
\hline RF transceiver power on $\left(T_{\text {on }}\right)(\mathrm{ms})$ & 2 \\
\hline Maximum critical data index $\left(\xi_{\max }\right)$ & 7 \\
\hline
\end{tabular}

TABLE 2: Numerical parameters.

capable of enhancing the system performance significantly. However, such benefits highly depend on the network topology and link quality.

4.3. Energy Consumption of TCMN. The throughput of the TCMN has been investigated in this section. The throughput 


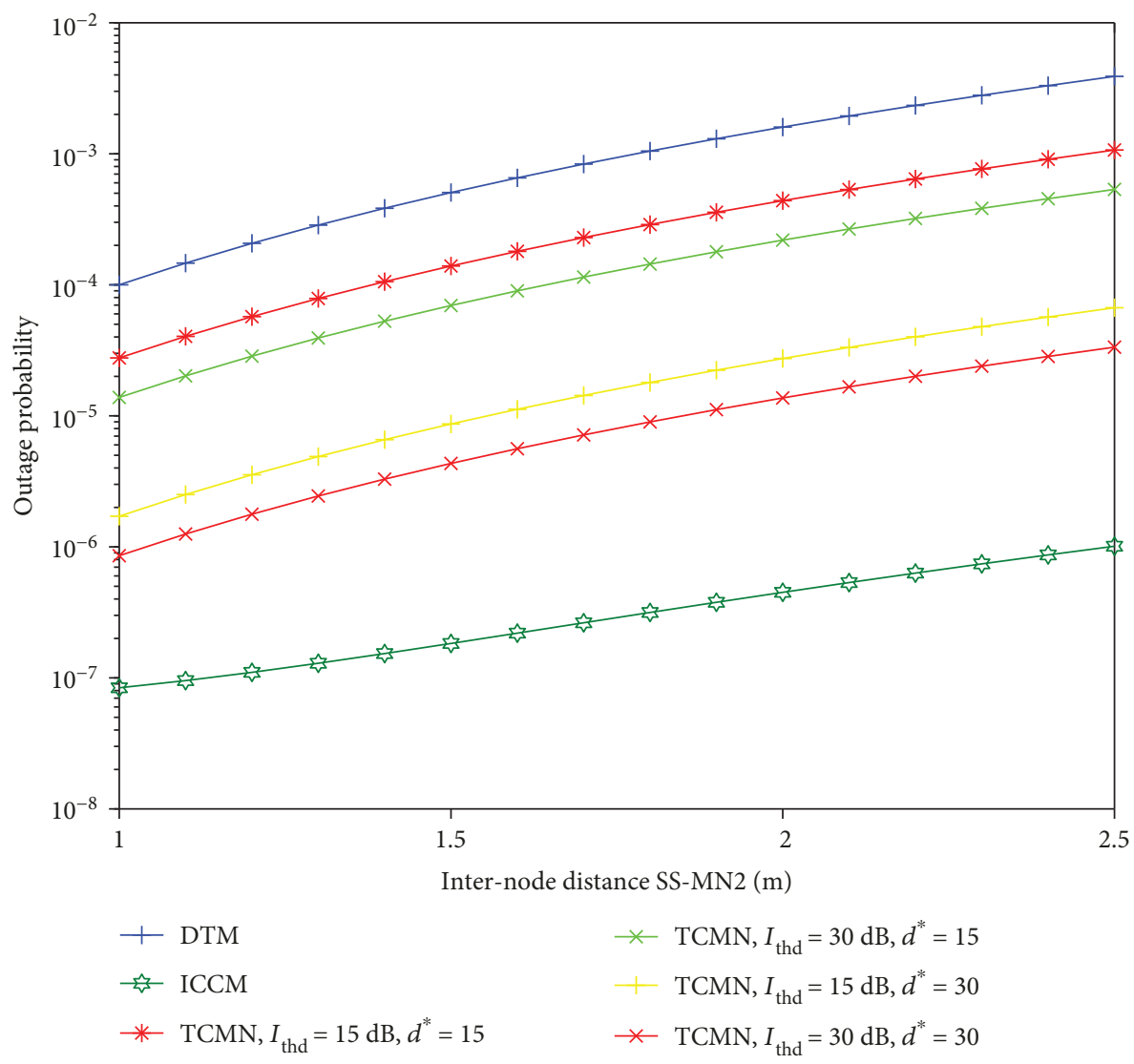

FIGURE 5: Comparison of outage probability of DTM, ICCM, and TCMN with internode distance of SS - MN2 link. In all cases, $\xi_{\text {min }}$ is 6 , $\rho$ is $4, P_{\mathrm{N}}$ is $-70 \mathrm{~dB}$, and $\delta_{\mathrm{c}}$ is 5 .

between $i$ and $j$ nodes of TCMN is expressed as follows [29]:

$$
\eta^{\mathrm{TCMN}}=\frac{E(P)}{T_{\mathrm{e} 2 \mathrm{e}}} R_{\max }[\mathrm{bps}]
$$

where $E(P)$ is average the transmission time for the payload bits. Let $L$ be the packet size (in bytes) and the data rate utilized in transmitting packet is $\eta^{\text {TCMN }}$. At the same time, the time to transmit one packet is $L / \eta^{\mathrm{TCMN}}$, and accordingly, the energy consumed at SS for transmitting one packet is $P_{\mathrm{ss}}\left(L / \eta^{\mathrm{TCMN}}\right)$ in which $P_{\mathrm{ss}}$ is the transmit power used by $P_{\mathrm{ss}}$ . As explained earlier, links of wireless network are prone to losing packets. Hence, it is possible that a packet will not reach the receiver until it is retransmitted several times. For this reason, we utilize $\varphi$ to represent the number of transmissions performed by SS for the same packet. Therefore, the energy consumed by SS to transmit a packet can be expressed as follows [30]:

$$
E_{\mathrm{TCMN}}=\varphi P_{\mathrm{ss}}\left(\frac{L}{\eta^{\mathrm{TCMN}}}\right)
$$

where $\varphi=1 / P_{\mathrm{CDT}-\mathrm{CMN}}$, and plugging (1) and (21) in (22), we obtain the energy consumed by SS using the TCMN protocol.

\section{Simulation and Discussion}

In our simulations, WBSN sensors and cellular users are colocated in the same cell and they distributed over a circle area with a radius of $300 \mathrm{~m}$, and the cellular base station is located in the cell center. Furthermore, an arbitrary topology has been taken into account, where sensors are arbitrarily distributed in $3.5 \times 3.5$ square area in $\mathrm{m}^{2}$; a number of the sensors are fixed in this area. The destination is located at the center of the human body area $(0,0)$, and the source is located at $\left(d_{\mathrm{s}, \mathrm{mn} 2}, 0\right)$. The numerical parameter used in this paper is given in Table 2. Figure 5 shows the comparison of outage probability of the DTM, ICCM, and TCMN as a function of $d_{\mathrm{s}, \mathrm{mn} 2}$. In general, the outage probability of the ICCM and TCMN achieves better performance compared to the DTM. But, we can see that the ICCM achieves better performance than TCMN due to interference limitation. Furthermore, the outage performance of the TCMN improves when WBSN sensors are located far away from cellular users (PR and PS). Similarly, the outage performance of the TCMN improves when interference threshold $\left(I_{\text {thd }}\right)$ is low. Based on the above results, we can derive the conclusion that TCMN improves when the WBSN sensors are located far away from the cellular users and the interference threshold is low. 


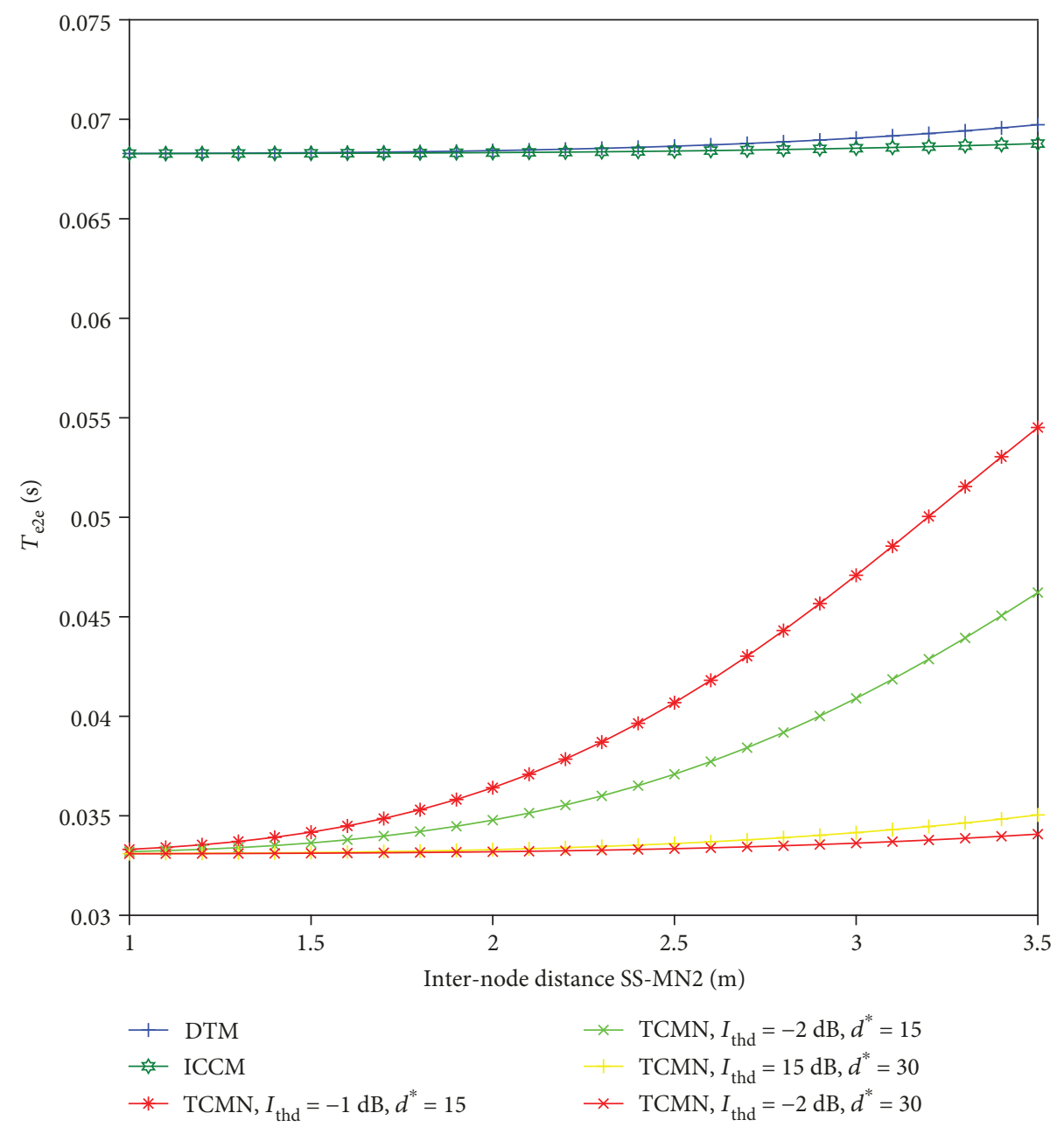

FIGURE 6: Comparison of e2e delay of DTM, ICCM, and TCMN with internode distance of SS - MN2 link. In all cases, $\xi_{\text {min }}$ is 6, $\rho$ is 4, and $P_{\mathrm{N}}$ is $-70 \mathrm{~dB}$.

Figure 6 shows the comparison of outage probability of the DTM, ICCM, and TCMN protocols as a function of $d_{\mathrm{s}, \mathrm{mn} 2}$. From the figure, we observed the following:

(1) Both the e2e delays of DTM and ICCM decrease and become similar to each other for the low $d_{\mathrm{s}, \mathrm{mn} 2}$. On the other hand, we can see that the e2e delay of ICCM is better than DTM when $d_{\mathrm{s}, \mathrm{mn} 2}$ is large

(2) However, the results show that the e2e delay of the TCMN protocol is less compared to the DTM and ICCM because the average time due to collision term is omitted according to (14)

(3) As expected, the e2e delay of the TCMN reduces when WBSN sensors are located far away from cellular users (PR and PS)

(4) The e2e delay of the TCMN reduces when interference threshold $\left(I_{\text {thd }}\right)$ is low

(5) The e2e delay of the TCMN is less with respect to DTM and ICCM by ratios $47 \%$ and $51.5 \%$, respectively
Figure 7 shows the comparison of throughput of the DTM, ICCM, and TCMN as a function of $d_{\mathrm{s}, \mathrm{mn} 2}$. We can notice from the figure that the throughput of the TCMN outperforms the DTM and ICCM. Furthermore, it can be seen from the results that the ICCM outperforms the DTM. The throughput of the proposed protocol increased with respect to DTM and ICCM by ratios $49 \%$ and $52 \%$, respectively.

Figure 8 shows the comparison of energy consumption of the DTM and ICCM protocols as a function of $d_{\mathrm{s}, \mathrm{mn} 2}$. It is observed from the figure that the ICCM achieves the lowest energy consumption, compared to the DTM. As expected, the energy consumption is reduced as the distance of SS MN2 is reduced. Furthermore, it can be seen from the results the energy saving of ICCM with respect to DTM by a ratio of $3 \%$.

Figure 9 shows the comparison of energy consumption of the TCMN as a function of $d_{\mathrm{s}, \mathrm{mn} 2}, I_{\text {thd }}$, and $d^{*}$. From the result, as $I_{\text {thd }}$ and $d^{*}$ grow, the energy consumption per sensor is reduced and vice versa. In addition, as the $d_{\mathrm{s}, \mathrm{mn} 2}$ increases, the energy consumption per sensor is 


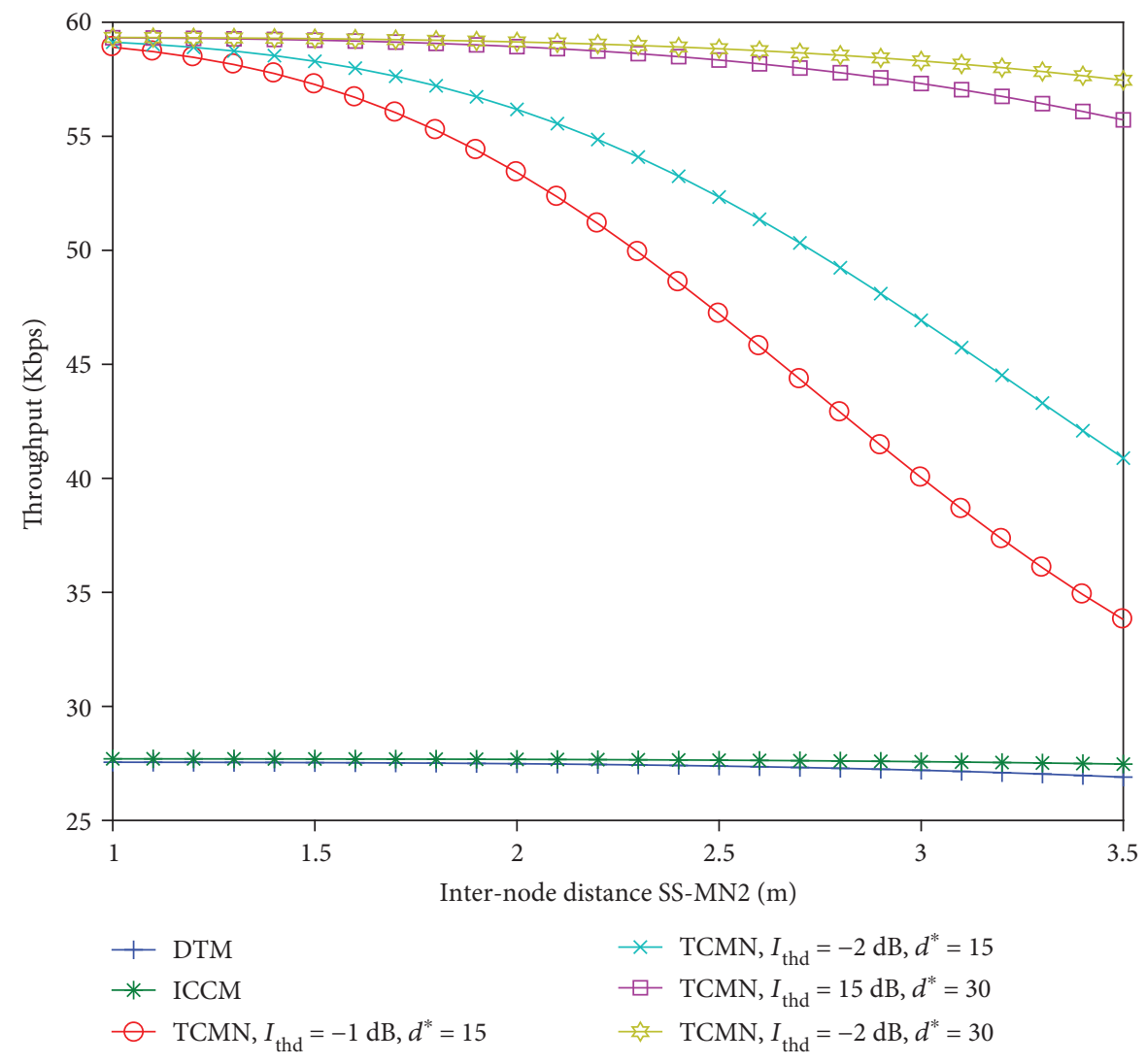

FIGURE 7: Comparison of throughput of DTM, ICCM, and TCMN with internode distance of SS - MN2 link. In all cases, $\xi_{\text {min }}$ is 6, $\rho$ is 4 , and $P_{\mathrm{N}}$ is $-70 \mathrm{~dB}$.

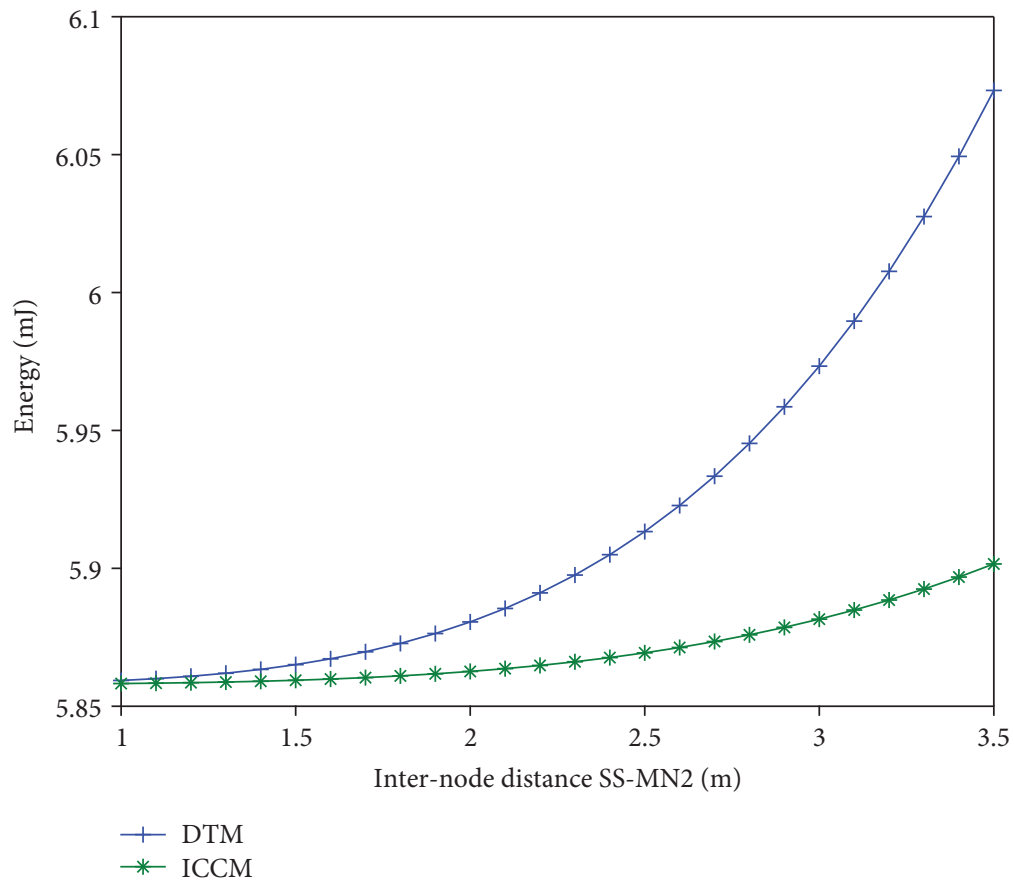

FIGURE 8: Comparison of energy consumption of DTM and ICCM with internode distance of SS - MN2 link. In all cases, $\xi_{\text {min }}$ is $6, \rho$ is 4 , and $P_{\mathrm{N}}$ is $-70 \mathrm{~dB}$. 


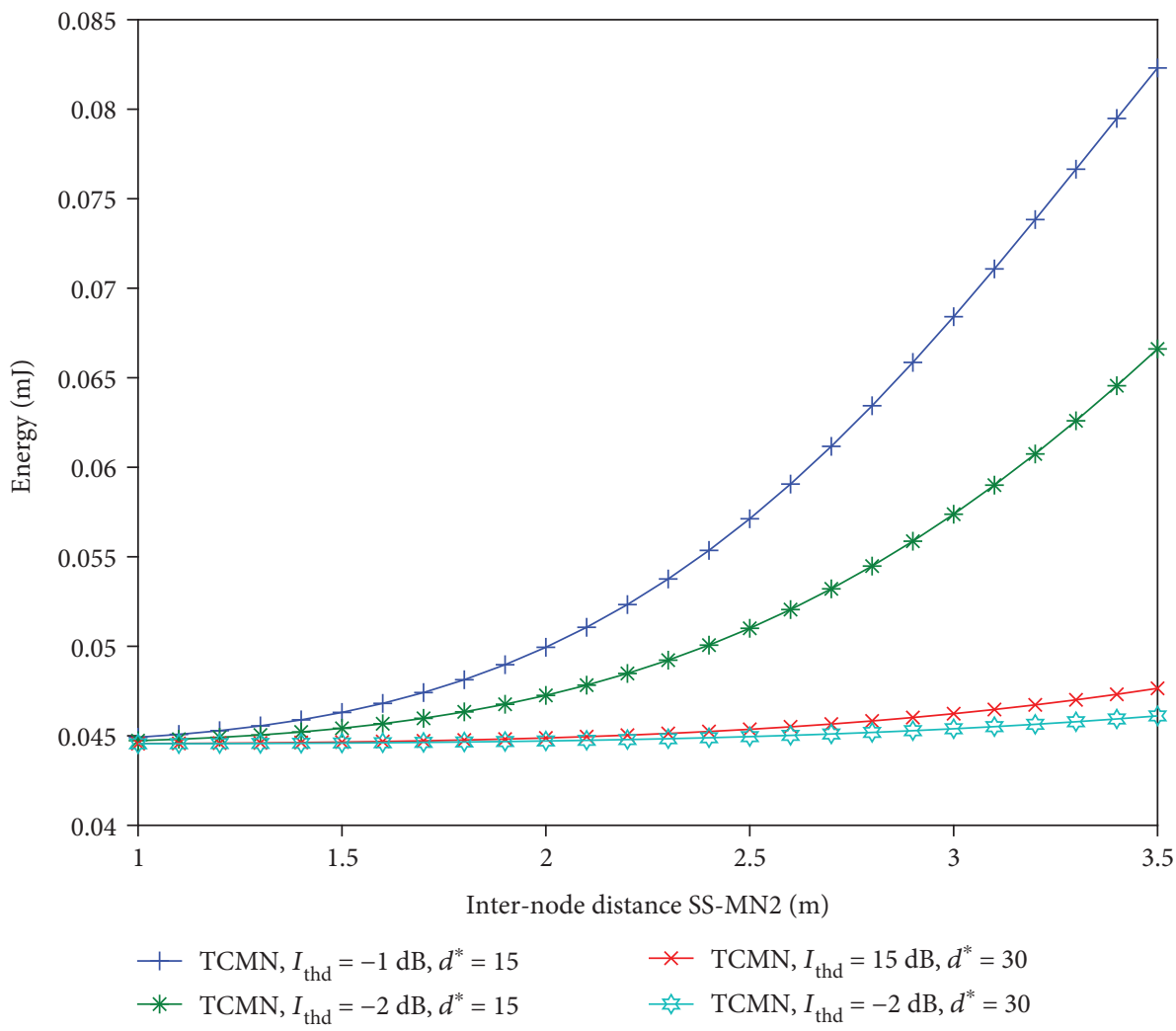

FIGURE 9: Comparison of energy consumption of TCMN with internode distance of SS - MN2 link. In all cases, $\xi_{\min }$ is 6, $\rho$ is 4 , and $P_{\mathrm{N}}$ is $-70 \mathrm{~dB}$.

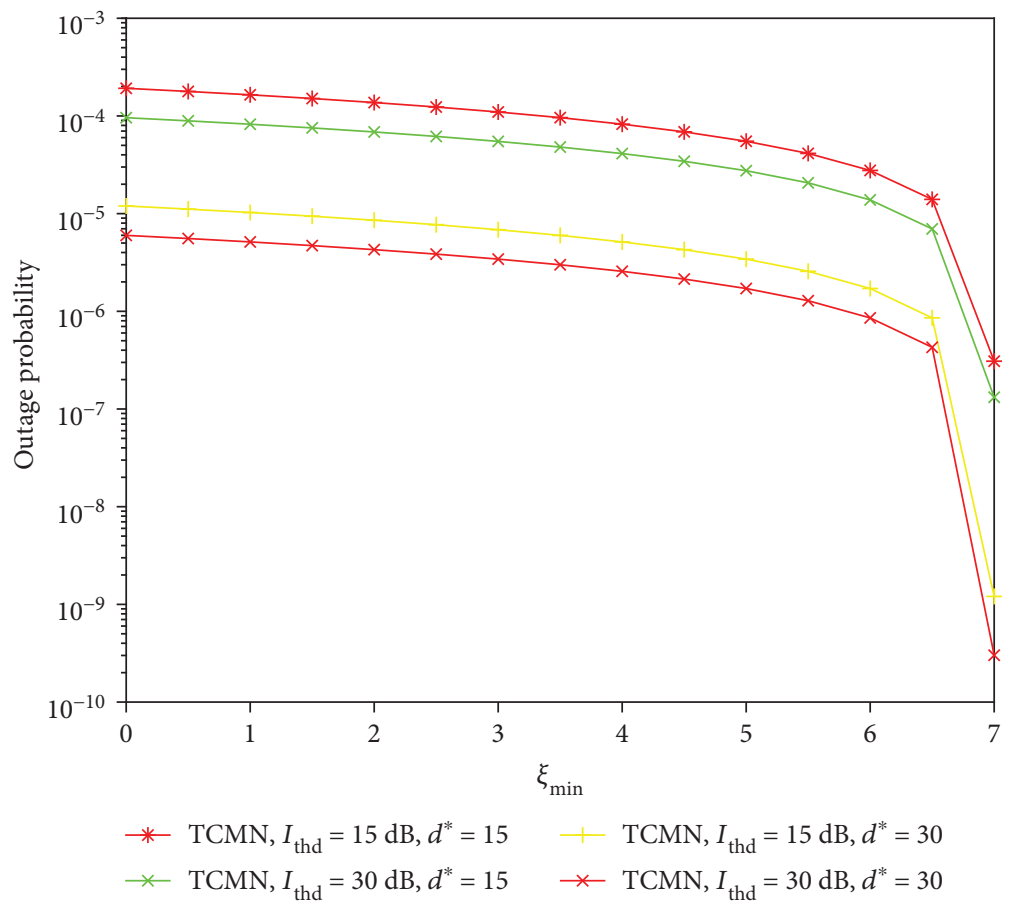

FIgURE 10: Comparison of outage probability of TCMN with $\xi_{\min }$. In all cases, $\rho$ is 4 and $P_{\mathrm{N}}$ is $-70 \mathrm{~dB}$. 


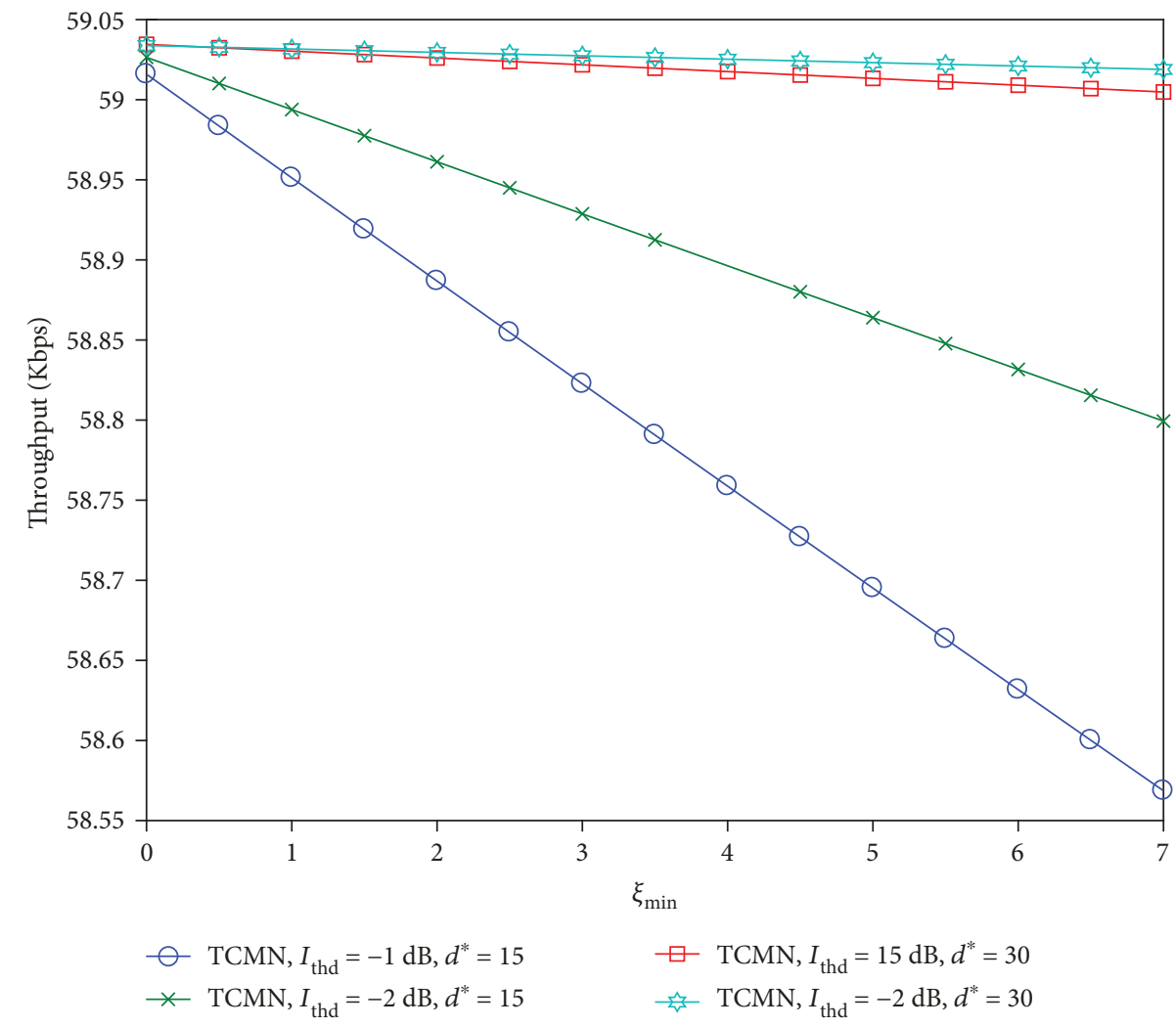

Figure 11: Comparison of throughput of TCMN with $\xi_{\min }$. In all cases, $\rho$ is 4 and $P_{\mathrm{N}}$ is $-70 \mathrm{~dB}$.

increases. Furthermore, it can be seen from the results the energy saving of TCMN with respect to ICCM and DTM by a ratio of $99 \%$.

The outage probability and throughput of the TCMN as a function of the $\xi_{\min }$ are plotted in Figures 10 and 11, respectively. The main objective of the proposed protocol is to deliver the critical data to the destination efficiently. Therefore, Figures 10 and 11 show that as the critical data index increases, the TCMN reduces the outage probability and increases the throughput to deliver the data efficiently.

\section{Conclusion}

In this paper, a novel cognitive cooperative communication with two master node protocols for WBSN is proposed that taken into consideration the nature of the collected data. The proposed protocol is based on the IEEE 802.15.6 CSMA policy under fading channel model and is named as TCMN. The objective of the proposed protocol can be summarized as follows: first, reduce the retransmission process and shift it to the master nodes; second, reduce the collision through utilizing licensed spectrum; and third, sensor node protocols remain the same because all the updates are done on the master nodes. The proposed protocol achieved a better transmission quality for the critical data. Furthermore, the TCMN can substantially reduce the outage probability, e2e delay, throughput, and energy consumption, compared to DTM IEEE 802.15.6 CSMA and ICCM. To this end, we have shown that the energy saving of the
TCMN is $99 \%$ with respect to DTM and ICCM under IEEE 802.15.6 CSMA. In future work, we will design and investigate a MAC protocol for inter-WBSN cooperation.

\section{Data Availability}

All data are available in the manuscript.

\section{Conflicts of Interest}

The authors declare that there are no conflicts of interest regarding the publication of this paper.

\section{References}

[1] P. Gope and T. Hwang, "Untraceable sensor movement in distributed IoT infrastructure," IEEE Sensors Journal, vol. 15, no. 9, pp. 5340-5348, 2015.

[2] P. Gope and T. Hwang, "A realistic lightweight authentication protocol preserving strong anonymity for securing RFID system," Computers \& Security, vol. 55, pp. 271-280, 2015.

[3] Y. Liao, M. Leeson, M. Higgins, and C. Bai, "Analysis of in-toout wireless body area network systems: towards QoS-aware health Internet of things applications," Electronics, vol. 5, no. 4 , p. $38,2016$.

[4] M. Ghamari, B. Janko, R. Sherratt, W. Harwin, R. Piechockic, and C. Soltanpur, "A survey on wireless body area networks for ehealthcare systems in residential environments," Sensors, vol. 16, no. 6, p. 831, 2016. 
[5] P. Gope and T. Hwang, "BSN-care: a secure IoT-based modern healthcare system using body sensor network," IEEE Sensors Journal, vol. 16, no. 5, pp. 1368-1376, 2016.

[6] S. M. R. Islam, D. Kwak, M. H. Kabir, M. Hossain, and K.S. Kwak, "The Internet of things for health care: a comprehensive survey," IEEE Access, vol. 3, pp. 678-708, 2015.

[7] Y. J. Fan, Y. H. Yin, L. D. Xu, Y. Zeng, and F. Wu, "IoT-based smart rehabilitation system," IEEE Transactions on Industrial Informatics, vol. 10, pp. 1568-1577, 2014.

[8] H. Moosavi and F. M. Bui, "Optimal relay selection and power control with quality-of-service provisioning in wireless body area networks," IEEE Transactions on Wireless Communications, vol. 15, no. 8, pp. 5497-5510, 2016.

[9] X. Chen, M. Ma, and A. Liu, "Dynamic power management and adaptive packet size selection for IoT in e-healthcare," Computers \& Electrical Engineering, vol. 65, pp. 357-375, 2018.

[10] A. Alkhayyat, O. Gazi, and S. B. Sadkhan, "The role of delay and connectivity in throughput reduction of cooperative decentralized wireless networks," Mathematical Problems in Engineering, vol. 2015, Article ID 294016, 10 pages, 2015.

[11] A. Alkhayyat, "Joint next-hop/relay selection for distributive multihop cooperative networks," Discrete Dynamics in Nature and Society, vol. 2015, Article ID 613168, 10 pages, 2015.

[12] K. Ho-Van, "Exact outage analysis of modified partial relay selection in cooperative cognitive networks under channel estimation errors," IET Communications, vol. 10, no. 2, pp. 219-226, 2016.

[13] J. Lee, H. Wang, J. G. Andrews, and D. Hong, "Outage probability of cognitive relay networks with interference constraints," IEEE Transactions on Wireless Communications, vol. 10, no. 2, pp. 390-395, 2011.

[14] F. Jingling, L. Wei, and L. Yang, "Performance enhancement of wireless body area network system combined with cognitive radio," in 2010 International Conference on Communications and Mobile Computing, vol. 3, pp. 313-317, Singapore, Singapore, 2010.

[15] M. Nazir and A. Sabah, "Cooperative cognitive WBSN: from game theory to population dynamism," in 2011 3rd International Congress on Ultra Modern Telecommunications and Control Systems and Workshops (ICUMT), pp. 1-6, Singapore, Singapore, 2011.

[16] R. Chavez-Santiago, K. E. Nolan, O. Holland et al., "Cognitive radio for medical body area networks using ultra wideband," IEEE Wireless Communications, vol. 19, no. 4, pp. 74-81, 2012.

[17] A. R. Syed and K.-L. A. Yau, "On cognitive radio-based wireless body area networks for medical applications," in 2013 IEEE Symposium on Computational Intelligence in Healthcare and e-health (CICARE), pp. 51-57, Singapore, Singapore, 2013.

[18] L. Januszkiewicz, "Simplified human body models for interference analysis in the cognitive radio for medical body area networks," in 2014 8th International Symposium on Medical Information and Communication Technology (ISMICT), pp. 1-5, Singapore, Singapore, 2014.

[19] A. Z. Shaikh and L. Tamil, "Cognitive radio enabled telemedicine system," Wireless Personal Communications, vol. 83, no. 1, pp. 765-778, 2015.

[20] M. Mahbub, S. M. R. Ali, Z. Iqbal, M. S. Kaiser, and M. S. Islam, "Performance analysis of cognitive cooperative communications for WBSN," in 2015 IEEE international conference on telecommunications and photonics (ICTP), pp. 1-5, Singapore, Singapore, 2015.

[21] S. Bhandari and S. Moh, "A survey of MAC protocols for cognitive radio body area networks," Sensors, vol. 15, no. 4, pp. 9189-9209, 2015.

[22] M. Bhatia and K. Kumar, "Network selection in cognitive radio enabled wireless body area networks," Digital Communications and Networks, vol. 3, 2018.

[23] S. Sodagari, S. Member, and B. Bozorgchami, "Technologies and challenges for cognitive radio enabled medical wireless body area networks," IEE Access, vol. 6, pp. 29567-29586, 2018.

[24] E. Pei, L. Li, W. Cheng, and J. Jiang, "An energy efficient spectrum sensing algorithm in EH-HCRSN," IEEE Access, vol. 6, pp. 63021-63032, 2018.

[25] H. Al-mishmish, D. Hammood, H. A. Rahim, and Q. H. Abbasi, "Critical data-based incremental cooperative communication for wireless body area network," Sensors, vol. 18, no. $11,2018$.

[26] F. Masud, A. H. Abdullah, G. Abdul-Salaam, and F. Ullah, "Traffic adaptive MAC protocols in wireless body area networks," Wireless Communications and Mobile Computing, vol. 2017, Article ID 8267162, 14 pages, 2017.

[27] M. Khalid, Y. Wang, I. Ra, and R. Sankar, "Two-relay-based cooperative MAC protocol for wireless ad hoc networks," IEEE Transactions on Vehicular Technology, vol. 60, no. 7, pp. 33613373, 2011.

[28] D. A. Hammood, H. A. Rahim, A. Alkhayyat, R. B. Ahmed, and Q. H. Abbasi, "Reliable emergency data transmission using transmission mode selection in wireless body area network," Cogent Engineering, vol. 5, no. 1, pp. 1-19, 2018.

[29] R. Hekmat, Ad-hoc Networks: Fundamental Properties and Network Topologies, Springer Science \& Business Media, 2006.

[30] X. Tian, Y.-H. Zhu, K. Chi, J. Liu, and D. Zhang, "Reliable and energy-efficient data forwarding in industrial wireless sensor networks," IEEE Systems Journal, vol. 11, no. 3, pp. 1424$1434,2017$. 


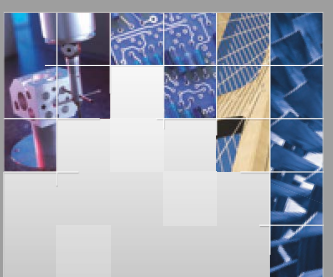

\section{Enfincering}
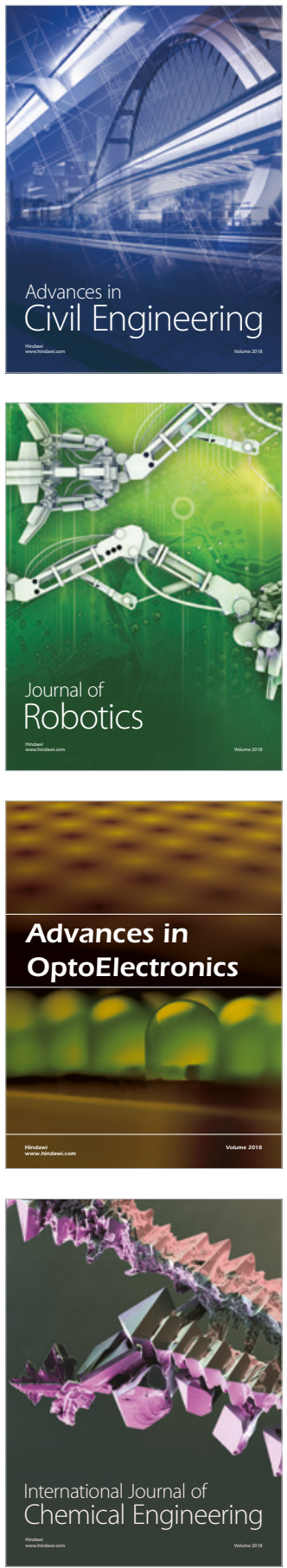

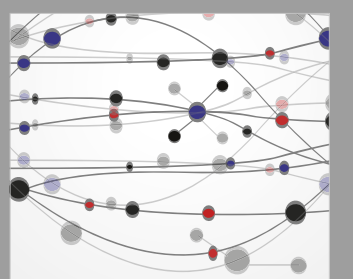

\section{Rotating \\ Machinery}

The Scientific World Journal

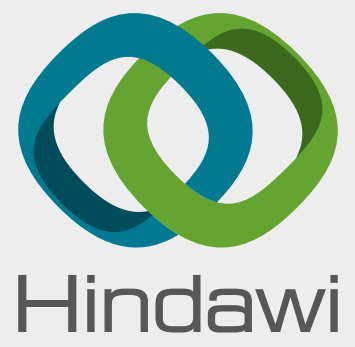

Submit your manuscripts at

www.hindawi.com
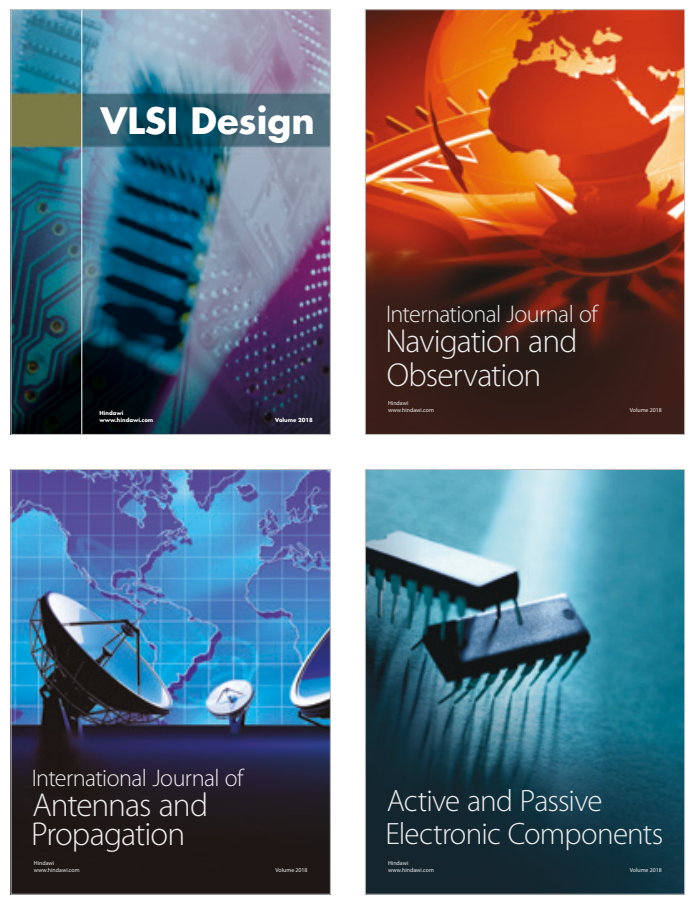
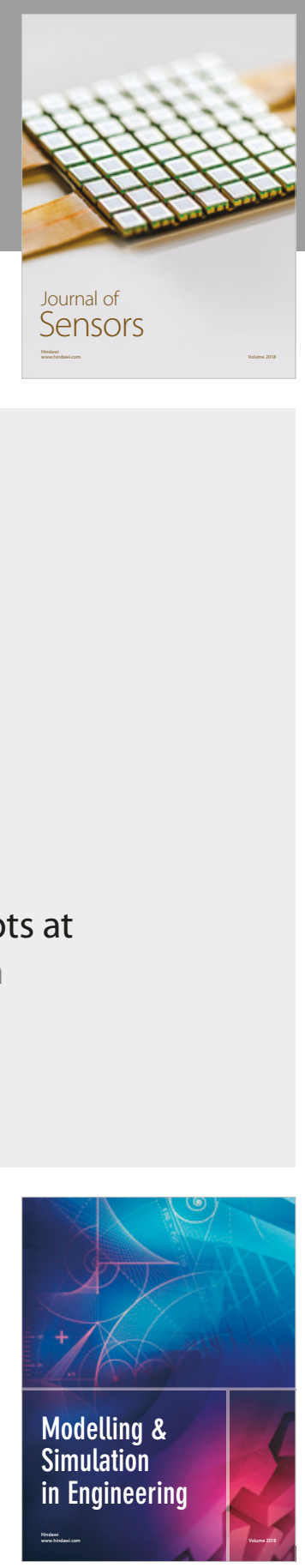

\section{Advances \\ Multimedia}
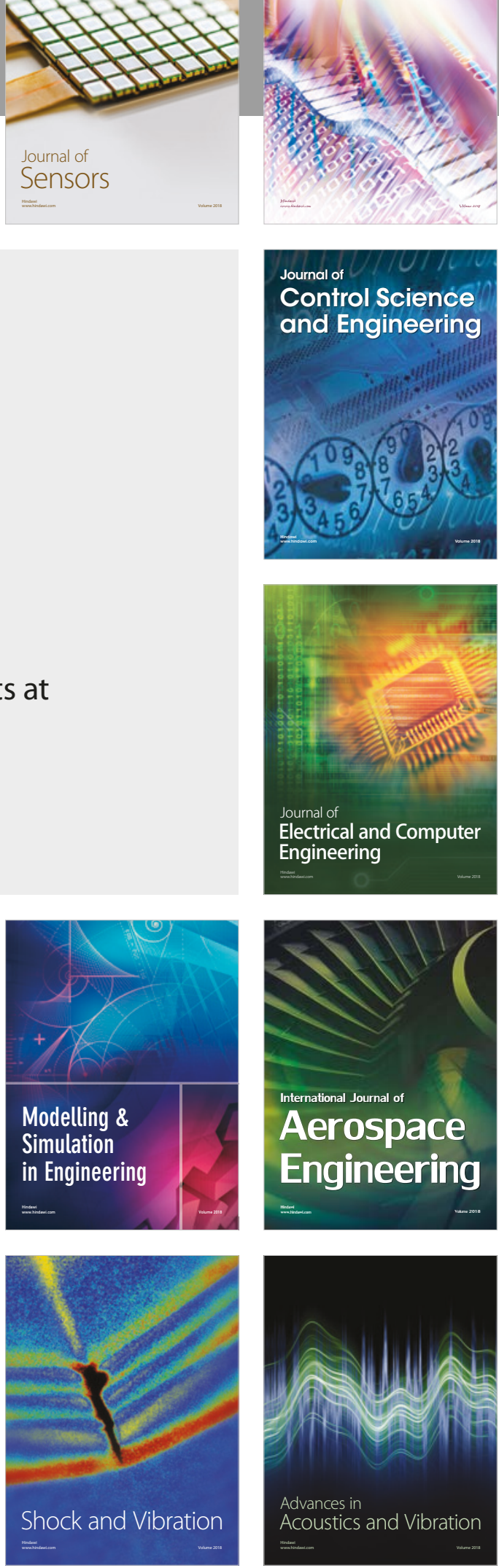